\title{
Electronic Coupling and Exciton Energy Transfer in CdTe Quantum-Dot Molecules
}

\author{
Rolf Koole, ${ }^{*}$ Peter Liljeroth, Celso de Mello Donegá, Daniël Vanmaekelbergh, and \\ Andries Meijerink
}

Contribution from the Debye Institute, Condensed Matter and Interfaces, Utrecht University, P.O. Box 80 000, 3508 TA Utrecht, The Netherlands

Received March 8, 2006; E-mail: r.koole@phys.uu.nl

\begin{abstract}
Stable dispersions of molecularlike aggregates of CdTe quantum dots are prepared by chemical cross-linking. Cryo-TEM images confirm the presence of cross-linked quantum dots and show that the size of the small aggregates can be controlled by the amount of cross-linker added. Optical measurements reveal two types of interdot interactions within these quantum-dot molecules: exciton energy transfer and electronic coupling. Quantitative information on the energy transfer rates in quantum-dot molecules is obtained by photoluminescence lifetime measurements. The degree of electronic coupling is dependent on the size of the quantum dots, which is supported by quantum mechanical calculations.
\end{abstract}

\section{Introduction}

Worldwide efforts in the bottom-up synthesis of colloidal semiconductor quantum dots (QDs) by wet-chemistry have led to highly luminescent nanocrystals of II-VI and III-V compounds with excellent control over the nanocrystal size, shape, and surface chemistry. ${ }^{1}$ Concomitantly, optical and electron tunneling spectroscopy, together with theoretical efforts, have led to an understanding of the energy level structure of nanocrystalline quantum dots. ${ }^{2,3}$ Strong confinement of the charge carriers in the nanocrystal host leads to discrete valence and conduction energy levels and an optical gap that is strongly size-dependent. Highly efficient photoluminescence and a photon energy that can be tailored by the nanocrystal size promise applications of semiconductor nanocrystals in optoelectrical devices such as LEDs, ${ }^{4,5}$ lasers, ${ }^{6,7}$ solar cells, ${ }^{8,9}$ and as optical labels in biomedical applications. ${ }^{10,11}$

Most of these applications are not based on isolated quantum dots but on a molecular or solidlike assembly of nanocrystals. It is obvious that the collective opto-electrical properties of quantum dot materials will be influenced by mutual interdot

(1) Yin, Y.; Alivisatos, A. P. Nature 2005, 437, 664.

(2) Bakkers, E. P. A. M.; Hens, Z.; Zunger, A.; Franceschetti, A.; Kouwenhoven, L. P.; Gurevich, L.; Vanmaekelbergh, D. Nano Lett. 2001, 1, 551. (3) Banin, U.; Millo, O. Annu. Rev. Phys. Chem. 2003, 54, 465.

(4) Tessler, N.; Medvedev, V.; Kazes, M.; Kan, S. H.; Banin, U. Science 2002, 295, 1506.

(5) Coe, S.; Woo, W. K.; Bawendi, M.; Bulovic, V. Nature 2002, 420, 800.

(6) Klimov, V. I.; Mikhailovsky, A. A.; Xu, S.; Malko, A.; Hollingsworth, J. A.; Leatherdale, C. A.; Eisler, H. J.; Bawendi, M. G. Science 2000, 290, 314.

(7) Wang, C. J.; Wehrenberg, B. L.; Woo, C. Y.; Guyot-Sionnest, P. J. Phys. Chem. B 2004, 108, 9027 .

(8) Nozik, A. J. Physica E 2002, 14, 115

(9) Gur, I.; Fromer, N. A.; Geier, M. L.; Alivisatos, A. P. Science 2005, 310 462 .

(10) Michalet, X.; Pinaud, F. F.; Bentolila, L. A.; Tsay, J. M.; Doose, S.; Li, J. J.; Sundaresan, G.; Wu, A. M.; Gambhir, S. S.; Weiss, S. Science 2005 $307,538$.

(11) Mulder, W. J. M.; Koole, R.; Brandwijk, R. J.; Storm, G.; Chin, P. T. K.; Strijkers, G. J.; de Mello Donegá, C.; Nicolay, K.; Griffioen, A. W. Nano Lett. 2006, 6, 1-6. interactions. Two important interactions, viz. exciton energy transfer and electronic coupling, have been investigated extensively in extended arrays of colloidal quantum dots (quantum dot solids $)^{12-19}$ but much less in small molecularlike assemblies. ${ }^{20-22}$ Electronic coupling in single pairs of epitaxially grown InAs/GaAs QDs was reported previously. ${ }^{23-25}$ Small assemblies consisting of only a few nanocrystal building blocks are of great interest in fundamental research. Electron and hole delocalization, spin-transfer, and entanglement are key aspects that could eventually lead to future applications such as quantum computing. ${ }^{23-29}$

(12) Kagan, C. R.; Murray, C. B.; Nirmal, M.; Bawendi, M. G. Phys. Rev. Lett. 1996, 76, 1517.

(13) Crooker, S. A.; Hollingsworth, J. A.; Tretiak, S.; Klimov, V. I. Phys. Rev Lett. 2002, 89, 186802/1.

(14) Achermann, M.; Petruska, M. A.; Crooker, S. A.; Klimov, V. I. J. Phys Chem. B 2003, 107, 13782 .

(15) Franzl, T.; Koktysh, D. S.; Klar, T. A.; Rogach, A. L.; Feldmann, J Gaponik, N. Appl. Phys. Lett. 2004, 84, 2904.

(16) Wuister, S. F.; Koole, R.; de Mello Donegá, C.; Meijerink, A. J. Phys. Chem. B 2005, 109, 5504 .

(17) Micic, O. I.; Ahrenkiel, S. P.; Nozik, A. J. Appl. Phys. Lett. 2001, 78, 4022.

(18) Artemyev, M. V.; Bibik, A. I.; Gurinovich, L. I.; Gaponenko, S. V.; Woggon, U. Phys. Rev. B: Condens. Matter Mater. Phys. 1999, 60, 1504

(19) Dollefeld, H.; Weller, H.; Eychmuller, A. J. Phys. Chem. B 2002, 106, 5604.

(20) Wargnier, R.; Baranov, A. V.; Maslov, V. G.; Stsiapura, V.; Artemyev, M.; Pluot, M.; Sukhanova, A.; Nabiev, I. Nano Lett. 2004, 4, 451.

(21) Ni, T.; Nagesha, D. K.; Robles, J.; Materer, N. F.; Mussig, S.; Kotov, N A. J. Am. Chem. Soc. 2002, 124, 3980.

(22) Ma, Q.; Su, X. G.; Wang, X. Y.; Wan, Y.; Wang, C. L.; Yang, B.; Jin, Q. H. Talanta 2005, 67, 1029.

(23) Bayer, M.; Hawrylak, P.; Hinzer, K.; Fafard, S.; Korkusinski, M. Wasilewski, Z. R.; Stern, O.; Forchel, A. Science 2001, 291, 451.

(24) Krenner, H. J.; Sabathil, M.; Clark, E. C.; Kress, A.; Schuh, D.; Bichler, M.; Abstreiter, G.; Finley, J. J. Phys. Rev. Lett. 2005, 94.

(25) Yamauchi, S.; Komori, K.; Morohashi, I.; Goshima, K.; Sugaya, T. J. Appl. Phys. 2006, 99.

(26) Ouyang, M.; Awschalom, D. D. Science 2003, 301, 1074.

(27) Oosterkamp, T. H.; Fujisawa, T.; van der Wiel, W. G.; Ishibashi, K. Hijman, R. V.; Tarucha, S.; Kouwenhoven, L. P. Nature 1998, 395, 873

(28) Franceschetti, A.; Zunger, A. Phys. Rev. B: Condens. Matter. Mater. Phys. 2001, 63, 153304/1.

(29) Loss, D.; DiVincenzo, D. P. Phys. Rev. A:At., Mol., Opt. Phys. 1998, 57, 120 . 


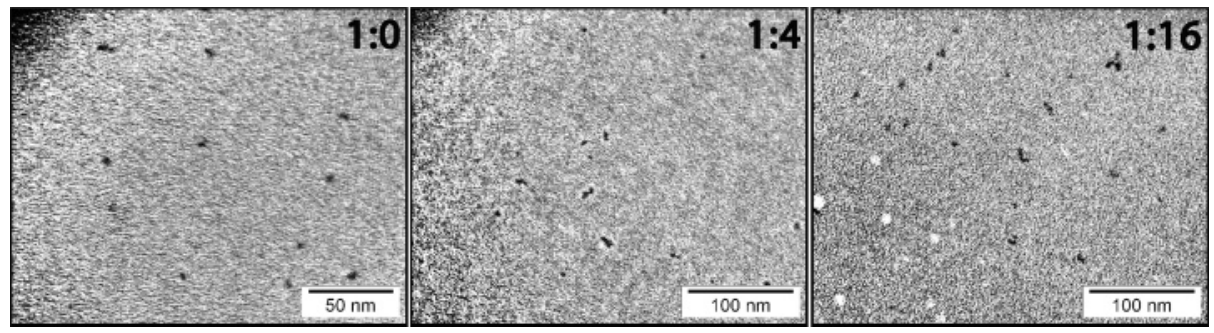

Figure 1. Cryo-TEM images of single gQDs (gQD/linker ratio 1:0) and of samples with a ratio of 1:4 and 1:8.

Although exciton energy transfer in small molecularlike aggregates consisting of only a few colloidal nanocrystals has been reported previously, electronic coupling in such systems has not been investigated. ${ }^{20,22,30}$ This is partly due to the fact that such systems are much more difficult to prepare than quantum dot solids. Although chemically blind dispersion forces are at work in the formation of nanocrystal superlattices, the formation of small quantum-dot molecules from nanocrystal building blocks requires well-controlled chemical linking. ${ }^{31}$

Here, we present a study of dispersed molecularlike aggregates of CdTe nanocrystals that are prepared by controlled cross-linking in solution. The structure of the aggregates is studied with Cryo-TEM. Electronic coupling and exciton energy transfer in these molecularlike aggregates is studied by absorption and (time-resolved) luminescence spectroscopy. Experimental evidence for electronic coupling is found, even at room temperature, and is supported by quantum mechanical calculations. Efficient energy transfer is studied by photoluminescence decay measurements, which show a clear distinction between single and cross-linked QDs in solution.

\section{Experimental Section}

Highly efficient $(\mathrm{QE}>50 \%)$ red $(\varnothing 5.1 \mathrm{~nm})$, yellow $(\varnothing 3.8 \mathrm{~nm})$, and green (Ø $3.4 \mathrm{~nm}$ ) emitting CdTe QDs (rQDs, yQDs, and gQDs) were synthesized following a literature procedure..$^{32}$ The dodecylaminecapped QDs obtained from the synthesis were recapped with allylamine (AA) by a ligand exchange. This was achieved by heating the QDs in an excess of AA for $4 \mathrm{~h}$ at $50{ }^{\circ} \mathrm{C}$. Subsequently, 1,6 hexanedithiol (HdT) was used to cross-link the QDs, because both thiol-groups of HdT can easily replace the allylamine-capping molecules that have a weaker interaction with the cadmium surface atoms of the CdTe QDs. Because the QDs were capped with the short AA molecules, a relatively short cross-linker (i.e., HdT) could be used to minimize the distance between the cross-linked QDs. By accurately varying the concentrations of cross-linker molecules in the QD dispersions, we were able to control the size of molecularlike aggregates of cross-linked CdTe QDs, which we will refer to as QD molecules from here. Several series were prepared and measured, which showed that the observed shifts in absorption and emission spectra as well as the decrease in emission intensities within one series are reproducible. To correct for the small variations in concentration within one series (less than 10\%), the emission spectrum of each sample was corrected according to the absolute value of the first absorption peak. We refer to the Supporting Information for more experimental details.

(30) Wang, S.; Mamedova, N.; Kotov, N. A.; Chen, W.; Studer, J. Nano Lett. 2002, $2,817$.

(31) Brousseau, L. C.; Novak, J. P.; Marinakos, S. M.; Feldheim, D. L. Adv Mater. 1999, 11, 447

(32) Wuister, S. F.; van Driel, F.; Meijerink, A. Phys. Chem. Chem. Phys. 2003, 5,1253 .

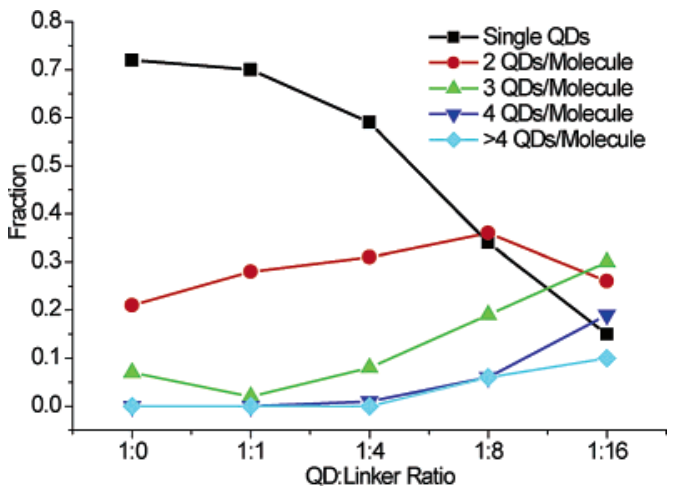

Figure 2. Fraction of QDs present as single QDs or present in QD molecules of different sizes for 5 different $\mathrm{QD} /$ linker ratios. The lines serve as a guide for the eye.

\section{Results and Discussion}

1. Cryo-TEM on QD Molecules. To obtain information about the size of the synthesized QD molecules, we performed cryo-TEM on the samples used for the optical measurements. Regular TEM was ill-suited for this purpose, because it is difficult to discriminate between cross-linked QDs and QDs that self-assemble on the TEM grid due to drying-effects. The cryoTEM images of three samples of the gQDs with different QD/ linker ratios are shown in Figure 1. As can be seen, mainly single QDs are present at a $\mathrm{QD} /$ linker ratio of 1:0, whereas aggregates of 2 and more QDs are visible at higher QD/linker ratios. We refer to the Supporting Information for more cryoTEM images of samples with these and other QD/linker ratios.

To obtain statistical information about the distribution in aggregate-size at a certain QD/linker ratio, the amount of QDs per aggregate was analyzed from the cryo-TEM images. For each QD/linker ratio, between 150 and 350 QDs were counted and grouped into QDs present as single QDs and in aggregates of two, three, etc. QDs per QD molecule. Despite the relatively low resolution inherent to cryo-TEM images, a reasonable statistical analysis was possible, the result of which is shown in Figure 2. As can be seen, the fraction of single QDs decreases significantly with increasing linker concentration. Note that a small fraction of QD molecules is already formed in the system without cross-linker molecules. The fraction of QD molecules with 2 QDs per molecule gradually increases up to the QD/ linker ratio of 1:8, after which it slightly decreases. The fraction of QD molecules with 3 or more QDs per molecule also increases at higher $\mathrm{QD} /$ linker ratios. For the highest $\mathrm{QD} /$ linker ratios $(>8)$, the solutions are turbid due to the formation of larger clusters. Because these large clusters may not be incorporated in the cryo-TEM film, the numbers obtained for these samples may not be representative for the solution. These results confirm 

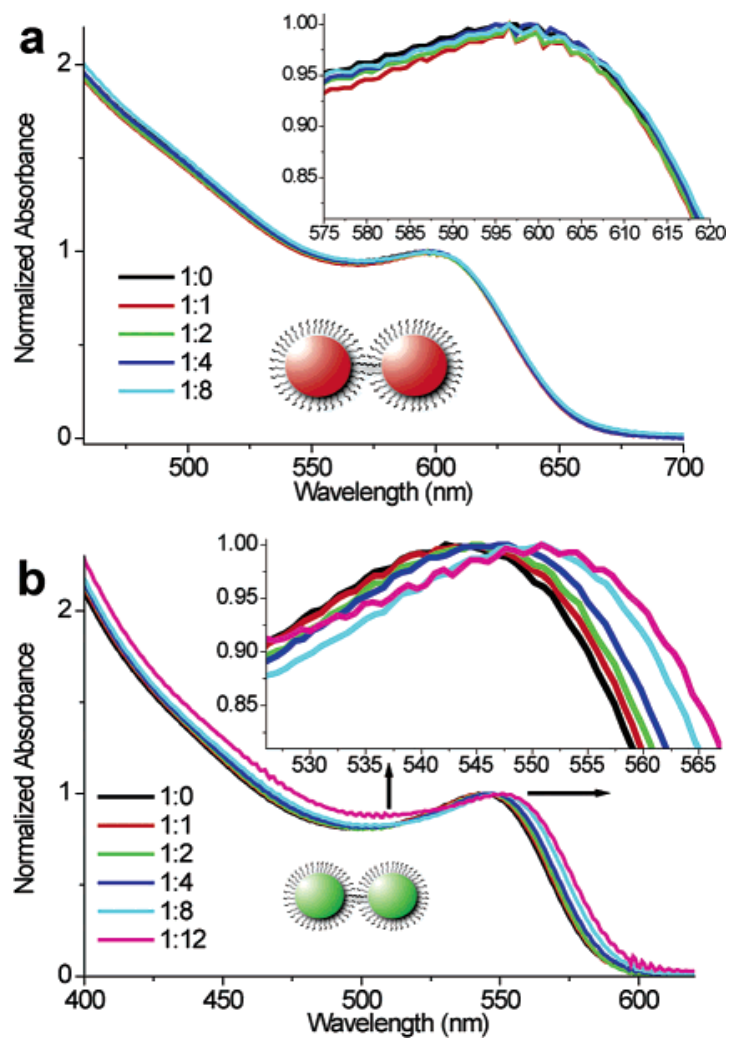

Figure 3. Normalized absorption spectra of cross-linked rQDs (a) and gQDs (b) in toluene at various QD/linker ratios. (Insets) Magnification of the first absorption peak.

the controllable cross-linkage of QDs by HdT, with an increase in both size and number of the aggregates at higher QD/linker ratios, and give an estimate for the fraction of QDs present in QD molecules for different QD/linker ratios.

2. Absorption and Emission Spectra. Figure 3a (3b) shows the normalized absorption spectra of solutions of cross-linked rQDs (gQDs) for different QD/linker ratios. As can be seen in Figure $3 \mathrm{a}$, the absorption spectra of the rQD molecules coincide after normalization. On the other hand, the gQD molecules show a clear, gradual red-shift for increasing QD/linker ratios (up to $40 \mathrm{meV}$ ). In addition, a broadening of the absorption spectrum is observed at the ratios $1: 8$ and $1: 12$, resulting in a less pronounced minimum at around $500 \mathrm{~nm}$. At higher linker concentrations, the dispersions of the QD molecules became turbid, and the absorption spectra were altered due to light scattering by these large aggregates (not shown).

Emission spectra of the same samples of rQDs and gQDs at different QD/linker ratios were also measured. At increasing QD/linker ratios, the rQD molecules (Figure 4a) show a gradual decrease in emission intensity, a red-shift of the emission peak (see inset) up to $52 \mathrm{meV}$, and an increase in the relative intensity of the defect-related emission at $\lambda>675 \mathrm{~nm}$ (see inset). Up to a QD/linker ratio of 1:8, the gQD molecules (Figure 4b) also show a gradual red-shift but no decrease in emission intensity, whereas the defect-related emission remains negligible (at $\lambda>$ $625 \mathrm{~nm}$ ). At ratios 1:16 and 1:32 (turbid solutions), a clear decrease in emission intensity, a further red-shift of the emission peak up to $74 \mathrm{meV}$, and an increase in defect-related emission is observed.

The results described above for the rQD molecules can be explained by exciton energy transfer (ET) due to dipole-dipole
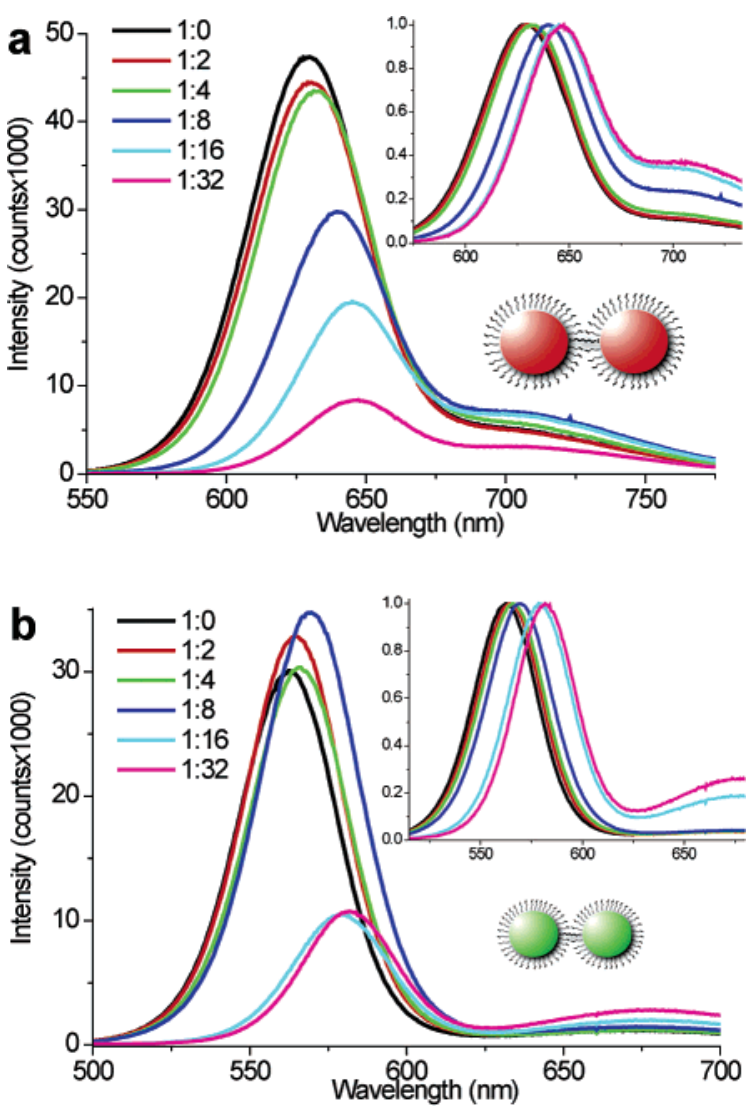

Figure 4. Emission spectra of cross-linked rQDs (a) and gQDs (b) in toluene at various QD/linker ratios. (Inset) Normalized spectra. Excitation is at $406 \mathrm{~nm}$.

interactions between the rQDs. ET has been reported before for QD-solids, ${ }^{12-16}$ where the migration of an exciton from higher-band gap QDs (donors) to smaller-band gap QDs or defect states (acceptors) resulted in a red-shift and decrease of the excitonic emission peak, together with an increase in defectrelated emission. No significant changes in the absorption spectra of the rQD molecules are observed, which indicates that quantum mechanical coupling between the rQD molecules is weak. Therefore, we conclude that the changes observed in the emission spectra of the rQD molecules (Figure 4a) can be accounted for solely by energy transfer. Photoluminescence decay measurements confirm this interpretation (vide infra).

The gQD molecules also show a red-shift in the excitonic emission peak. In contrast to the rQD molecules, this is accompanied by a red-shift in the absorbance. Furthermore, no decrease in excitonic emission intensity is observed up to a QD/ linker ratio of 1:8 whereas the relative intensity of the defectrelated emission remains constant and very weak. These results, which we reproducibly measured on gQDs from separate syntheses, cannot be explained by ET. The observations can be qualitatively explained by a hybridization of the band edge orbitals (i.e., $1 \mathrm{~S}_{\mathrm{e}}$ and $1 \mathrm{~S}_{\mathrm{h}}$ ) of the individual QDs due to a strong electronic (tunneling) coupling between neighboring gQDs. The resulting reduction of the confinement energy due to electron (and hole) delocalization explains the red-shift and broadening of the absorption spectra of the gQD molecules (Figure $3 b$ ). ${ }^{33,34}$

(33) Bryant, G. W.; Jaskolski, W. Physica E 2002, 13, 293.

(34) Jaskolski, W.; Bryant, G. W.; Planelles, J.; Zielinski, M. Int. J. Quantum Chem. 2002, 90, 1075. 


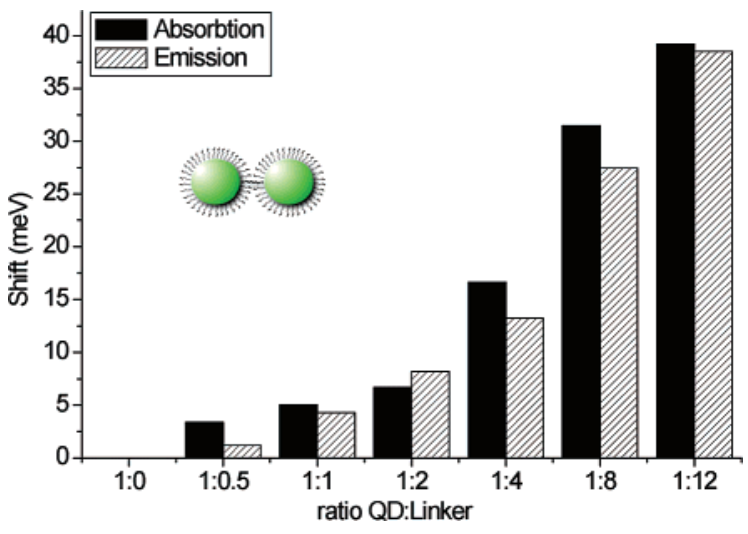

Figure 5. Comparison between the red-shift in first absorption peak (solid bars) and excitonic emission peak (striped bars) at various $\mathrm{gQD} /$ linker ratios.

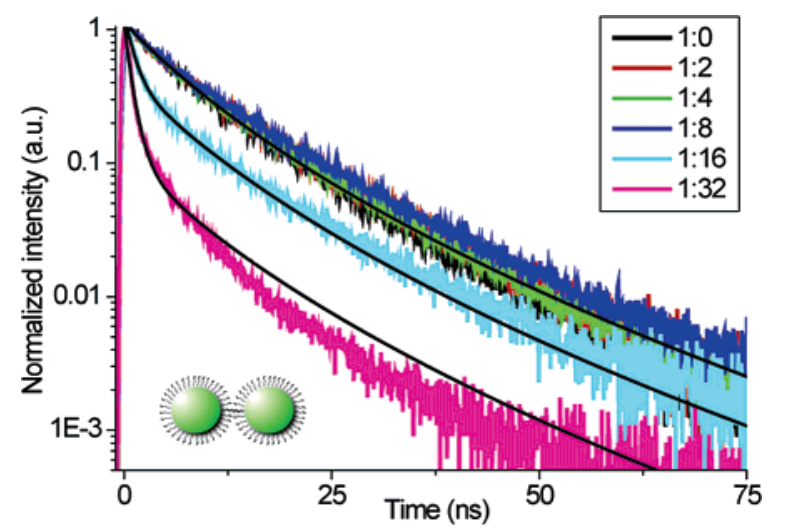

Figure 6. Photoluminescence decay curves of gQDs at various QD/linker ratios, measured at $540 \mathrm{~nm}$. Solid black curves show the results of the fitting (see text).

Similar observations have been reported previously for QDsolids of extremely small CdS, CdSe, and InP nanocrystals. ${ }^{17-19}$ The degree of electronic coupling between the dots is expected to be stronger for smaller QDs. The extension of the evanescent wave function of the carriers outside the QD is significantly larger for smaller QDs, and hence, the hybridization of the energy levels is only observed for the smaller gQDs (a more quantitative consideration is presented below). Because the redshift in absorbance is caused by electronic coupling of the states involved in the lowest optical transitions of the individual gQDs, a comparable shift is expected for the excitonic emission peak. Figure 5 shows that there is indeed a similar gradual shift of the first absorption and excitonic emission peak of the $\mathrm{gQD}$ molecules at increasing $\mathrm{QD} /$ linker ratios. At the $\mathrm{QD} /$ linker ratios 1:16 and 1:32 (turbid solutions), a significant quenching and further red-shift of the excitonic emission peak is observed, together with an increase in defect-related emission (Figure 4b). Apparently, ET is absent in small gQD molecules and is observed in larger gQD molecules (consisting of more than two QDs). This is explained as follows: in the small gQD molecules, electronic coupling causes a delocalization of the charge carriers over the QD molecule and energy transfer cannot occur. In the larger gQD molecules (formed at QD/linker ratios higher than $1: 8$ ), the delocalization does not extend over the entire molecule, and ET can occur between a higher-energy domain and a lowerenergy domain within one aggregate.

In addition to the rQD molecules and gQD molecules, we have also cross-linked intermediate sized yellow emitting QDs
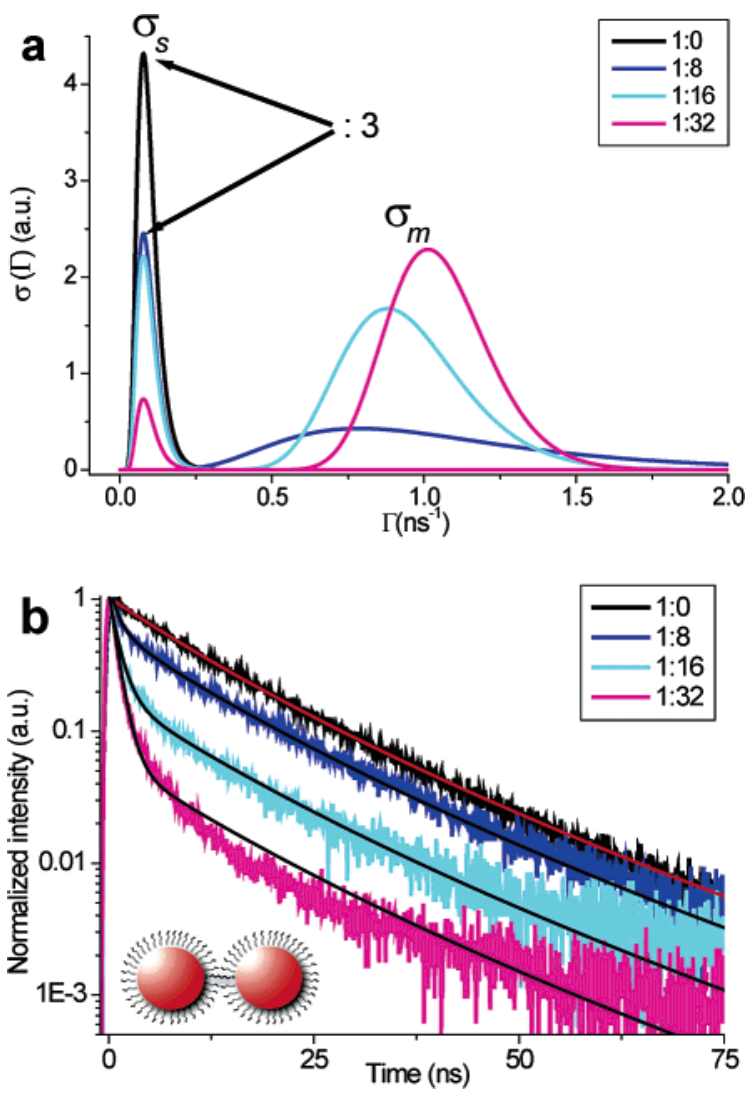

Figure 7. (a) Distribution functions of the decay rate of single rQDs $\left(\sigma_{\mathrm{s}}\right)$ and $\mathrm{rQD}$ molecules $\left(\sigma_{\mathrm{m}}\right)$ at different $\mathrm{QD} /$ linker ratios. Note that the curves of $\sigma_{\mathrm{s}}$ at ratios 1:0 and 1:8 were divided by three. (b) Photoluminescence decay curves of rQDs at various QD/linker ratios, measured at $580 \mathrm{~nm}$. Solid curves show the results of the fitting.

(yQDs, $3.8 \mathrm{~nm}$, excitonic emission peak at $590 \mathrm{~nm}$ ). The resulting yQD molecules show very similar results as compared to the gQD molecules: a red-shift in both absorption and emission spectra up to $\mathrm{QD} /$ linker ratio of $1: 12$, without a decrease in emission intensity (see Supporting Information). The red-shift in the absorption peak of these yQD molecules (up to $14 \mathrm{meV}$ ) is significantly smaller than for the gQD molecules (up to $40 \mathrm{meV}$ ), and larger than for the rQD molecules (no shift). The observed intermediate electronic coupling in the $\mathrm{YQD}$ molecules provides additional evidence for the size-dependent electronic coupling in QD molecules as described above.

3. Time-Resolved Photoluminescence Measurements. The photoluminescence decay curves of the single QDs and QD molecules were measured to investigate the exciton energy transfer process in more detail. The decay curves of the gQDs (Figure 6) were measured at a wavelength of $540 \mathrm{~nm}$, which is on the far blue side of the emission spectrum. Consequently, the exciton lifetimes of the large band gap QDs in the gQD molecules, which should act as donors in the case of ET, were probed. As can be seen in Figure 6, the gQDs show a nearly monoexponential decay up to a $\mathrm{QD} /$ linker ratio of 1:8. At higher $\mathrm{QD} /$ linker ratios, the decay curves become nonexponential, with a rapid initial decay. The fast initial luminescence decays for these largest $\mathrm{gQD}$ molecules within the first $\sim 2 \mathrm{~ns}$ is a clear signature of ET and is observed only for those samples that show a decrease and red-shift of the excitonic emission peaks (see Figure 4b). Further evidence for the presence of energy transfer is obtained from measurements of the decay curves as 

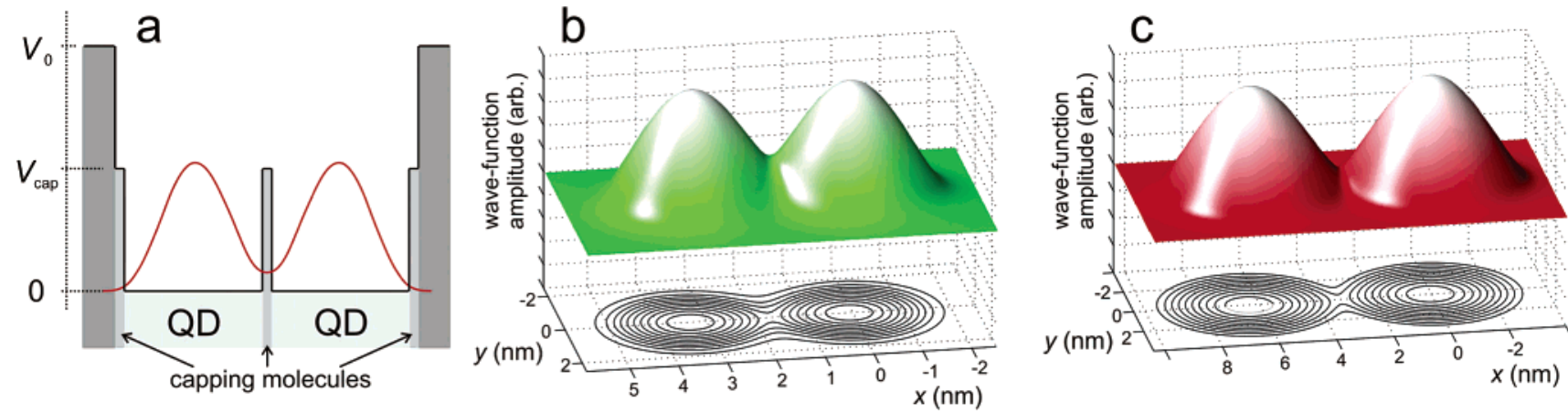

Figure 8. (a) Schematic representation of the energy diagram used as a basis for the calculations; (b) and (c) contour-plot and amplitude of the electron wave functions in two neighboring QDs with a diameter of $3 \mathrm{~nm}$ (b) and $6 \mathrm{~nm}$ (c). ( $V_{\text {cap }}=1.5 \mathrm{eV}, V_{0}=4 \mathrm{eV}$ ).

a function of wavelength. For systems in which energy transfer occurs (fast initial decay measured on the blue side of the spectrum), a feeding is observed for the decay curves measured for the acceptor emission on the red side of the spectrum due to energy transfer from the donors to the acceptors. It is wellknown that the ET rates in QD solids can range from $50 \mathrm{ps}^{-1}$ up to $\sim 2 \mathrm{~ns}^{-1}$, depending on the spectral overlap between donor and acceptor, the interdot distance, and dipole moment of the transitions involved. ${ }^{13,14,35}$ Importantly, the fingerprint of ET (i.e., fast initial decay) is not present in the decay curves of the $\mathrm{gQD}$ molecules up to a QD/linker ratio of 1:8. Because there is no evidence of ET in these $\mathrm{gQD}$ molecules, we ascribe the redshift in the emission and absorption spectra to a reduction in the optical gap due to electronic coupling.

To obtain more quantitative information on energy transfer rates from the lifetime measurements, we fitted the decay curves using a biexponential function. This implies a distinction between two radiative systems: QDs that are part of a QD molecule, and single QDs in solution. We ascribe the fast initial decay to the emission of those QDs that are part of a QD molecule, of which the total decay rate $\left(\Gamma_{\mathrm{m}}\right)$ is the sum of the radiative decay rate $\left(\Gamma_{\mathrm{rad}}\right)$ and the energy transfer rate $\left(\Gamma_{\mathrm{ET}}\right)$. The subsequent slow decay is attributed to the single QDs, because it has a similar decay in the long-time regime as measured for the QDs without cross-linker (1:0). In addition, a distribution function $(\sigma)$ was introduced for the decay rates of the single QDs $\left(\sigma_{\mathrm{s}}\right)$ and QD molecules $\left(\sigma_{\mathrm{m}}\right)$, to allow for a variation in the decay rates within one system. ${ }^{36}$ The fitting procedure is discussed in more detail in the Supporting Information. The resulting fits of the decay curves for the $\mathrm{gQD}$ molecules and rQD molecules are depicted as solid black curves in Figure 6 and Figure 7b, respectively. As can be seen in Figure $7 \mathrm{~b}$, a biexponential decay and, hence, ET is also observed for the $\mathrm{rQD}$ molecules at those $\mathrm{QD} /$ linker ratios where a clear decrease in intensity and a red-shift of the excitonic emission peak was observed (see Figure $4 b$ ).

Figure 7a shows the normalized distribution functions obtained from fitting the decay curves of the $\mathrm{QDD}$ molecules (Figure 7b). It can be seen that the distribution functions of the single rQDs $\left(\sigma_{\mathrm{s}}\right)$ and the rQD molecules $\left(\sigma_{\mathrm{m}}\right)$ are clearly separated, which confirms that they can be treated as two distinct systems. The average decay rate (maximum of the distribution function) of the single QDs ( $\left.\Gamma_{\text {rad }}\right)$ is found to be $0.08 \mathrm{~ns}^{-1}$ (13 ns). The decay rate of the rQD molecules increases at higher

(35) Förster, T. Naturwissenshaften 1946, 33, 166.

(36) James, D. R.; Ware, W. R. Chem. Phys. Lett. 1986, 126, 7.
QD/linker ratios, and has an average value of about $1.0 \mathrm{~ns}^{-1}$, which implies an energy transfer rate $\left(\Gamma_{\mathrm{ET}}\right)$ of $\sim 0.9 \mathrm{~ns}^{-1}$. The area of $\sigma_{\mathrm{s}}$ represents the fraction of single rQDs, and as can be seen, the fraction of single rQDs decreases with increasing QD/ linker ratio. Similarly, the fraction of QDs that are part of a QD molecule increases at increasing QD/linker ratios. For example, at a $\mathrm{QD} /$ linker ratio of 1:16, the fraction of single QDs is found to be $18 \%$, so there is still a significant amount of single QDs present.

4. Calculation of the Electronic Coupling Energy. In the green emitting QD molecules, the red-shift in absorbance and emission spectra can be as large as $40 \mathrm{meV}$ (see Figure 5). To see whether this can be explained by electronic coupling, we performed quantum mechanical calculations on two coupled CdTe QDs using an effective mass approach. The aim was to calculate the coupling energy, which we defined as the decrease in energy of the lowest conduction band level $\left(1 \mathrm{~S}_{\mathrm{e}}\right)$, and hence the reduction of the optical gap. We assumed that the $\pi$-bond in the allylamine ligands can reduce the effective tunneling barrier between the QDs, enhancing electronic coupling. Therefore, the calculation considered two spherical potential wells surrounded by a shell with a barrier height of $V_{\text {cap }}$ (the capping molecules, thickness $0.3 \mathrm{~nm}$ ) embedded in higher barrier matrix ( $V_{0}=4 \mathrm{eV}$, the solvent). The corresponding energy diagram is shown in Figure 8a. We solved the Schrödinger equation for a single electron $\left(m_{\mathrm{e}}=0.096\right)$ for the two lowest energy levels, which result from the hybridization of the lowest conduction band levels $\left(1 \mathrm{~S}_{\mathrm{e}}\right)$ of the isolated quantum dots. For reasons of simplicity, we neglect the coupling of the valence band levels, because the coupling energy is small due to the high effective hole mass $\left(m_{\mathrm{h}}\right)$ of 0.4 . The amplitude of the ground-state wave function (bonding molecular orbital) is shown in Figure $8 \mathrm{~b}$ and c for QDs with diameters of 3 and $6 \mathrm{~nm}$, respectively. The coupling is seen as the overlap of the wave functions of the individual QDs; there is a clear difference between the small and large coupled QDs.

Figure 9 shows the dependence of the coupling energy on the size of the QDs, assuming a distance of $0.4 \mathrm{~nm}$ between the QDs, and a value of $1.5 \mathrm{eV}$ for $V_{\text {cap }}$. The magnitude of the coupling energy for the gQD molecules $(\varnothing 3.4 \mathrm{~nm})$ of $20 \mathrm{meV}$ is in fairly good agreement with our experiments. Furthermore, Figure 9 confirms the qualitative expectation that small QDs couple more strongly than larger QDs. For the rQD molecules (Ø $5.1 \mathrm{~nm}$ ), the coupling energy is calculated to be $5 \mathrm{meV}$, which might explain that within our experimental resolution a reduction of the optical gap could not be observed. However, 


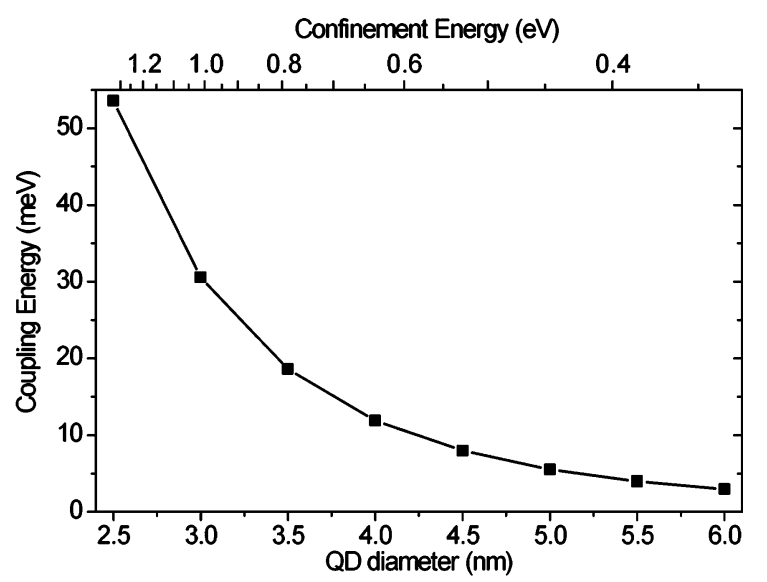

Figure 9. Calculated coupling energies of two coupled QDs as a function of the QD-diameter (bottom $x$-axis) and as a function of the confinement energy (top $x$-axis). The interdot distance was set at $0.4 \mathrm{~nm}, V_{\text {cap }}$ at $1.5 \mathrm{eV}$, and $V_{0}$ at $4 \mathrm{eV}$.

if the coupling energy is plotted against the calculated confinement energy of the individual QD (upper $x$-axis Figure 9), the difference in coupling energy between the gQD molecules $\left(E_{\text {conf }}\right.$ $=0.78 \mathrm{eV}$, derived from first absorption peak) and rQD molecules $\left(E_{\text {conf }}=0.57 \mathrm{eV}\right)$ is smaller. This discrepancy is caused by the overestimation of the confinement energy by the effective mass approximation. We also performed calculations varying the values for $V_{\text {cap }}$ and $V_{0}$, the interdot distance, and a situation where the influence of the capping molecule was neglected $\left(V_{\text {cap }}=V_{0}\right)$. The results all show similar trends, with coupling energies of tens of meV for the smaller gQDs and several meV for the rQDs (Supporting Information).

\section{Conclusions}

In conclusion, we have investigated the interactions in homonuclear QD molecules of red, yellow, and green emitting CdTe QDs. For the red emitting QD molecules, absorbance and emission spectra as well as the photoluminescence decay curves clearly show the presence of energy transfer, which causes the migration of excitons from higher to lower band gap rQDs. Information about the ET rates $\left(\sim 0.9 \mathrm{~ns}^{-1}\right)$ and the fraction of single QDs versus QD molecules were obtained by a biexponential fit of the decay curves. In contrast, the smaller gQD molecules show a similar red-shift in both absorption and emission spectra that cannot be explained by ET, which is supported by the photoluminescence decay curves. We propose that electronic coupling of the band edge orbitals from neighboring gQDs is responsible for the observed reduction of the band gap. The degree of electronic coupling is dependent on the extension of the wave function outside the QDs, which is larger for the smaller QDs. The size dependence of the coupling energy is confirmed by the results for yQD molecules consisting of intermediate sized yellow emitting QDs. For these QDs, an intermediate coupling energy of $14 \mathrm{meV}$ is found between the values for the gQD molecules (40 meV) and rQD molecules ( 0 $\mathrm{meV})$. Calculated coupling energies using an effective mass approach are in fair agreement with the experimentally obtained values and also confirm that the degree of coupling is dependent on the QD size.

Acknowledgment. We thank Hans Meeldijk (Electron Microscopy Utrecht, Department of Cell Biology) for all his efforts concerning the (cryo-)TEM-images, Bob Luigjes for the synthesis of the yellow QDs, and Floris van Driel for his support regarding the fitting procedures. Financial support from the European Union networks "FULLSPECTRUM" (SES6-CT2003-502620) and "NANOSPECTRA" (HPRH-CT-2001-00320) is gratefully acknowledged.

Supporting Information Available: Experimental details, Cryo-TEM images, absorption and emission spectra of the yQD molecules, details on the fitting procedure of the decay curves, and additional calculations of the coupling energy. This material is available free of charge via the Internet at http://pubs.acs.org.

JA061608W 